\title{
The impact of the type of operation on the parameters of a shunting diesel locomotive with hybrid power plant
}

\author{
Anatoliy Falendysh ${ }^{1}$, Mykyta Volodarets $^{1}$, Olha Kletska $^{1, *}$, and Viktoriia Hatchenko ${ }^{2}$ \\ ${ }^{1}$ Ukrainian State University of Railway Transport, Department of Rolling Stock Operation and Maintenance, \\ 7 Feuerbach square, 61050 Kharkiv, Ukraine \\ ${ }^{2}$ State University infrastructure and technologies, Traction rolling stock of railways Department, Kyiv, Ukraine
}

\begin{abstract}
In designing a traction vehicle it is necessary to take into account the type of field operation it is meant for. It was found out that during shunting operation a ChME3 diesel locomotive is in idling mode for almost half of its general running time. That is why, the introduction of a less powerful engine along with an energy storage device for shunting operations at a shunting diesel locomotive is appropriate. The calculations were made using a modernized shunting diesel locomotive ChME3 with hybrid transmission of power as an example. The dependences of the general running time of a diesel locomotive on the position of the engine driver controller under performing shunting, transportation and hump operation were given in percentages. For every operation mode there was calculated the optimum power of a diesel-generator plant and the optimum energy capacity of an energy storage system taking into account the above-mentioned dependences. It was found out that due to the introduction of a hybrid drive for the section discussed above and the corresponding mass of a train the aggregate fuel consumption will be reduced by $25 \%$ and the efficiency of travel will increase by one third.
\end{abstract}

\section{Introduction}

When designing a traction vehicle, it is necessary to take into account the type of operation for which it is intended. From this point of view, it would be expedient to consider this issue taking as an example the shunting diesel locomotive, because it performs a wide range of jobs. Determination of the parameters of the hybrid diesel locomotive is considered in [1-9], but they have a number of shortcomings, namely: cost indicators, or massdimensional, or indicators of operation and repair, or a set of these indicators are not taken into account.

Consider, for example, a modernized ChME3 locomotive with hybrid power transmission of determining the parameters of a hybrid vehicle, depending on the type of work performed: maneuvering, on the hill and on the soles.

\section{Research and discussion of the results}

It is necessary to analyze the work of the diesel generator set (DGS) during the entire working cycle, taking into account both the permanent and the transient modes during acceleration and braking. In this case, the importance of the ratio of constant time and transients throughout the work cycle is of great importance. It was suggested in [10] that under the operating cycle there was a certain set of stable and transitional modes of operation of a diesel engine (DG) by a thermal engine during a relatively short (several minutes) segment of time, which was repeated throughout the period a particular technological operation.

In an operational cycle not only the ratio of the separate modes, but also their sequence, duration and frequency of changes are reflected. The conditional operational cycle is also the subject of research in the estimated way. In the case of compilation of the conditional operational cycle lasting for about 11 minutes long-term, research of the DGS shunting locomotive (SL) operational modes conducted by the Ural unit of the AllRussian Scientific Research Institute of Railway Transport, was considered. The number of changes of the modes (switching of the position of the locomotive controller (PLC)) and the relative time of stay of DGS shunting locomotive on each PLC corresponds to an average value of these variables in maintenance. For this cycle, the lifetime of the diesel engines of the ChME3 of position of the locomotive controller was developed for various types of work (shunting operation, work on the hill and export work), which can be expressed by the appropriate massifs $\tau_{1 P L C}\left(\tau_{1 P L C i}\right), \tau_{2 P L C}\left(\tau_{2 P L C i}\right), \tau_{3 P L C}\left(\tau_{3 P L C i}\right)$, $i=0 \ldots 8$. In this case, $\Sigma\left(\tau_{1 P L C i}\right)=\Sigma\left(\tau_{2 P L C i}\right)=\Sigma\left(\tau_{3 P L C i}\right)=1$. In the case of the execution of shunting operation, we have a massif

$$
\tau_{1 P L C}=(45,5 ; 4,9 ; 17,5 ; 17,5 ; 8,8 ; 4,1 ; 1,2 ; 0,3 ; 0,2),
$$

for the corresponding positions of the controller of the driver,

"Corresponding author: gurao@ukr.net 


$$
\tau_{P L C}=(0 ; 1 ; 2 ; 3 ; 4 ; 5 ; 6 ; 7 ; 8) .
$$

The analysis has shown that nearly half of the overall operating time the locomotive works in idling mode. $N e_{\text {arly }} 50 \%$ of time work from a position, the first on the fourth. Thus, most of the time the locomotive works in the uneconomical mode. That is introduction on shunting locomotive of the engine of smaller power together with accumulator of energy (AE) for shunting work expediently.

Dependence of power consumption $\mathrm{AE}$ with the corresponding restrictions and cost indexes of modernization from DGS power for performance of shunting work (Fig. 1) it is made taking into account a technique, which is presented in [11].

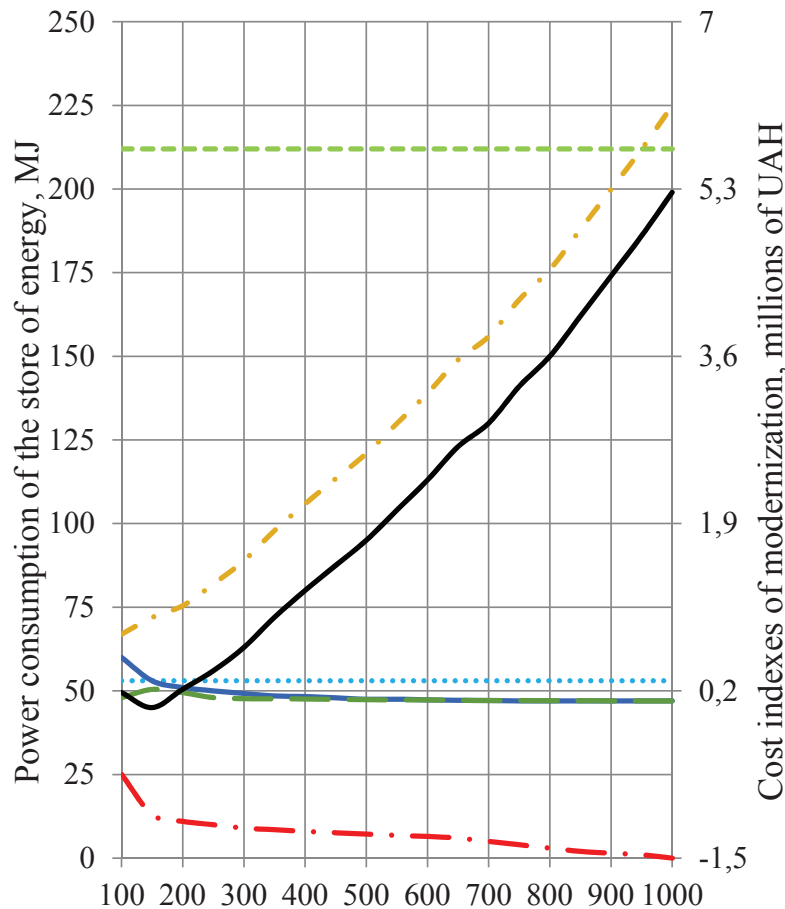

Power of the power plant, which is projected, $\mathrm{kW}$

- - Dependence of Ene on DG power

....... Restrictions of NE on weight, MJ

$\cdots$ Restrictions of NE on volume, MJ

- - Dependence of cost of DG on its power

— Dependence of cost of NE on DG power

— Dependence of economy of fuel on power

— Dependence of efficiency of modernization on DG power

Fig. 1. Dependence of power consumption of the store with the corresponding restrictions and cost indexes of modernization from diesel generator power for performance of shunting work

From this drawing, it is obvious that the optimum power of DGS for the considered operating mode of a locomotive is about $150 \mathrm{~kW}$ and power consumption NE - about 20 MJ. The period of operation of the diesel on PLC for operational cycle shunting locomotive of the
ChME3 series which works at a hump yard within one hour can be expressed through the massif,

$$
\tau_{2 P L C}=(54,8 ; 2,3 ; 9,8 ; 13,4 ; 13,0 ; 4,5 ; 0,9 ; 0,6 ; 0,6) .
$$

This cycle has been received in [10] by processing and synthesis of data about the operation of shunting locomotive of this type on various railroads of Ukraine. In this cycle of work more than half of the total time the locomotive is in idling mode and more than $40 \%$ of time works with the first on the fifth PLC, that is most of the time the locomotive works in the uneconomical mode.

Similarly, with shunting work, there is dependence of power consumption $\mathrm{AE}$ with the corresponding restrictions and cost indexes of modernization from DGS power for performance of work on hill (Fig. 2).

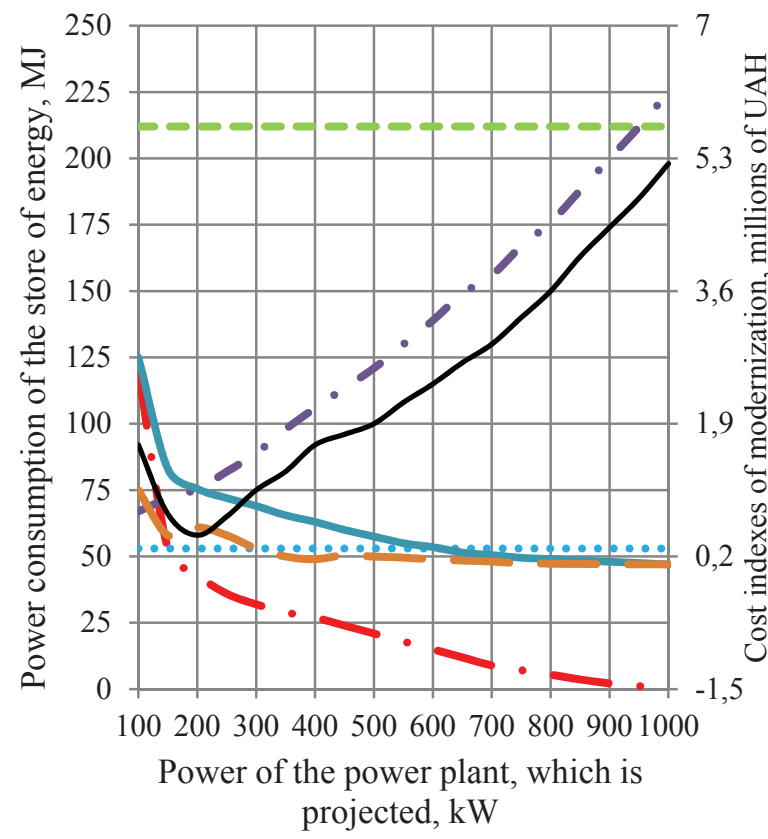

- Dependence of Ene on DG power

$\ldots$ Restrictions of NE on weight, MJ

- Restrictions of NE on volume, MJ

- - Dependence of cost of DG on its power

Dependence of cost of NE on DG power

- Dependence of economy of fuel on power

- Dependence of efficiency of modernization on DG power

Fig. 2. Dependence of power consumption of the store with the corresponding restrictions and cost indexes of modernization from diesel generator power for performance of work on the hill.

It follows from the calculations that the optimum power of DGS for the considered operating mode of a locomotive is about $200 \mathrm{~kW}$, and the necessary power consumption not is about $50 \mathrm{MJ}$. These parameters correspond to the smallest general expenses connected with modernization. 
The period of operation of the diesel on PLC for operational cycle of shunting locomotive ChME3 which performs export work can be expressed through the massif,

$$
\tau_{3 P L C}=(44,1 ; 1,7 ; 5,4 ; 6,6 ; 9,5 ; 19,3 ; 10,4 ; 2,3 ; 0,8) .
$$

When performing export work for about $44 \%$ of the time the locomotive is in the idling mode, and about $40 \%$ of the time works on 4-6 PLC, i.e. a considerable part of time the locomotive works in an uneconomical mode. For this cycle we have carried out calculations of parameters of a hybrid locomotive and the results are given in the Fig. 3.

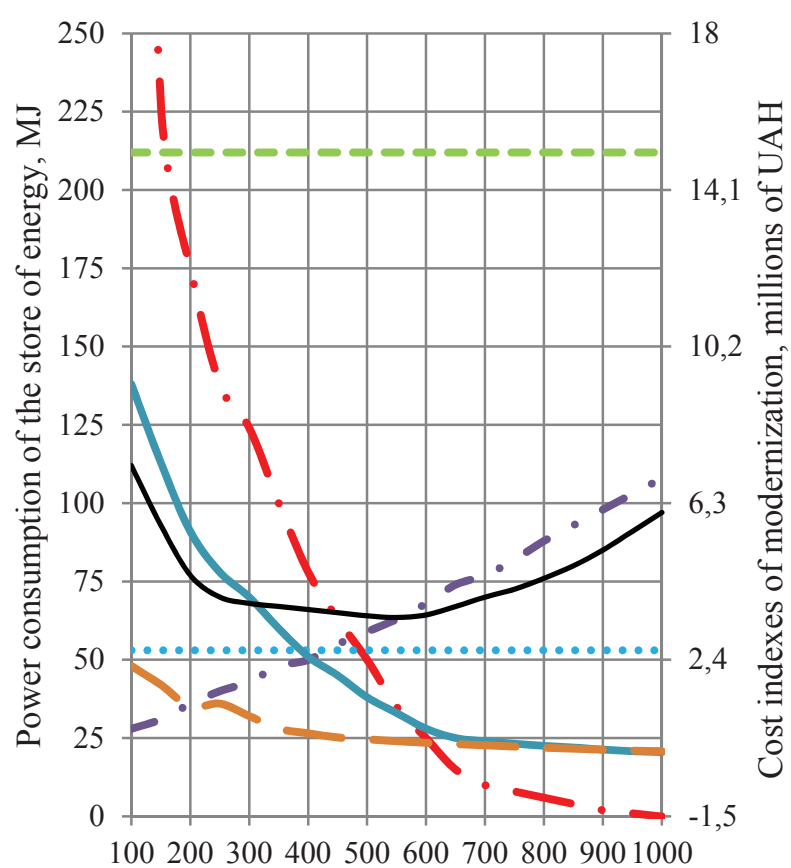

Power of the power plant, which is projected, $\mathrm{kW}$

- Dependence of Ene on DG power

.... Restrictions of NE on weight, MJ

- Restrictions of NE on volume, MJ

- - Dependence of cost of DG on its power

Dependence of cost of NE on DG power

- Dependence of economy of fuel on power

Dependence of efficiency of modernization on DG power

Fig. 3. Dependence of power consumption of the store with the corresponding restrictions and cost indexes of modernization from diesel generator power for performance of export work.

As a result of the approximation of the calculated values of power consumption AE Ene and power of DGS Neng it is revealed that generally with the size of reliability of approximation bigger 0,99 it is characterized by the following dependence, MJ,

$$
E_{n e}=D\left(\frac{1}{N_{\text {eng }}}\right)^{2}-E\left(\frac{1}{N_{\text {eng }}}\right)^{2}+F\left(\frac{1}{N_{\text {eng }}}\right)-G,
$$

where $D, E, F, G$ - the parameters characterizing a look carried out works and service conditions of the locomotive.

For each type of works the following dependences are obtained:

- for shunting work

$$
\begin{aligned}
& E_{n e}=243163\left(\frac{1}{N_{\text {eng }}}\right)^{2}-51028\left(\frac{1}{N_{\text {eng }}}\right)^{2}+ \\
& +3465,4\left(\frac{1}{N_{\text {eng }}}\right)-4,4354,
\end{aligned}
$$

- for work on the hill

$$
\begin{aligned}
& E_{n e}=10^{8}\left(\frac{1}{N_{\text {eng }}}\right)^{2}-2 \cdot 10^{6}\left(\frac{1}{N_{\text {eng }}}\right)^{2}+ \\
& +21818\left(\frac{1}{N_{\text {eng }}}\right)-17,489,
\end{aligned}
$$

- for export work

$$
\begin{aligned}
& E_{n e}=10^{8}\left(\frac{1}{N_{\text {eng }}}\right)^{2}-4 \cdot 10^{6}\left(\frac{1}{N_{\text {eng }}}\right)^{2}+ \\
& +70590\left(\frac{1}{N_{\text {eng }}}\right)-76,296 .
\end{aligned}
$$

The obtained dependences are fair for an interval of change of power of the power plant $N_{\text {eng }} \in[45 \ldots$ 993] where the lower restriction characterizes minimum necessary power of DGS for ensuring its own needs and start-off from the place of the locomotive in case refuses $\mathrm{Ne}$ and also considers extreme values of the DGS and AE mass-dimensional parameters. The top restriction is characterized by the most necessary power of DGS for performance of a certain type of works (undertakes the equal power of a locomotive of the ChME3 series which is taken as a basis). For the reviewed examples taking into account [11] and dependences (1-4) for dependence of the general expenses connected with modernization, Uzag, MJ, we have:

$$
\begin{aligned}
& U_{\text {zag }} N_{\text {eng }}=A N_{\text {eng }}^{2}+B N_{\text {eng }_{j}}+C+ \\
& +u_{2}\left[D\left(\frac{1}{N_{\text {eng }}}\right)^{2}-E\left(\frac{1}{N_{\text {eng }}}\right)^{2}+F\left(\frac{1}{N_{\text {eng }}}\right)-G\right]-U_{0}- \\
& -\left[\sum_{i=1}^{n}\left(N f_{i} g e_{0} \frac{\Delta \tau}{3600}\right)-\sum_{i=1}^{n} G_{i, j}\right] c t+ \\
& +C \sigma\left(H N_{\text {eng }_{j}}+K-1\right) \longrightarrow \min
\end{aligned}
$$

where $A, B, C, D, E, F, G, H, K$ - the coefficients characterizing the type of chosen DGS, type of work, service conditions and system of service and repair of a locomotive; 
$U_{0}$ - the liquidating cost of the basic diesel generator, $\mathrm{UAH}$;

$N f_{i}$, - the power of the power plant, $\mathrm{kW}$;

$g e_{0}$ - specific fuel consumption the diesel of the basic locomotive, $\mathrm{kg} / \mathrm{kW} \mathrm{h}$;

$\Delta \tau$ - a power differentiation interval during time, with;

$G_{i, j}$ - fuel consumption of a hybrid locomotive, $\mathrm{kg}$;

$c t$ - cost of fuel, UAH/kg;

$C \sigma-$ expenses for maintenance and repair of a basic locomotive, UAH.

Function (5) is a compound function, which includes functions continuous over a certain interval and discrete function. Therefore, in an analytical form it is not possible to receive optimum value of power $N_{\text {eng. }}$. It is rational also to approximate the determined function of dependence of $G\left(N_{\text {eng }}\right)$ as it does not allow to receive a simple universal formula for further calculations, therefore, fuel consumption considerably depends on the type of work performed, areas of operation, type and parameters of the AE hybrid locomotive. To obtain the needed level of reliability of approximation it is necessary to introduce a large number of additional parameters and too complicates function.

Therefore, the method of complete search as simple and such was applied to the solution of an objective of non-linear programming that yields quite exact results of computation. Its main shortcoming is that it requires a search of a large number of combinations, and it takes a lot of time for obtaining the decision. Therefore, target function of $U_{z a g}\left(N_{e n g}\right)$ was most simplified and given to one independent variable, taking into account the restrictions superimposed on it allowed to reduce considerably quantity of combinations of search and duration of computation.

As a result of the calculations we have chosen the corresponding parameters of hybrid $N_{\text {engopt }}$ locomotives the optimum power of DGS and $E_{n e}$ - optimum power consumption $\mathrm{AE}$ depending on the type of work performed, namely

- for shunting: $N_{\text {engopt }}=150 \mathrm{~kW} ; E_{n e}=20 \mathrm{MJ}$;

- for work on the hill: $N_{\text {engopt }}=200 \mathrm{~kW} ; E_{n e}=50 \mathrm{MJ}$;

- for export work: $N_{\text {engopt }}=530 \mathrm{~kW} ; E_{\text {ne }}=50 \mathrm{MJ}$.

Similar calculations have been carried out also for another type of hybrid drive of a locomotive with which the main source of energy is AE, and DGS is necessary only to resupply energy in AE (the advantage of this type of the drive is the fact that low-power DGS works most of the time in an economical operating mode. Depending on the type of work performed the following $E_{n e}$ and $N_{\text {engopt }}$ values are obtained:

- for shunting: $E_{n e}=370 \mathrm{MJ} ; N_{\text {engopt }}=50 \mathrm{~kW}$;

- for work on the hill: $E_{n e}=560 \mathrm{MJ} ; N_{\text {engopt }}=80 \mathrm{~kW}$;

- for export work: $E_{n e}=1300 \mathrm{MJ} ; N_{\text {engopt }}=100 \mathrm{~kW}$.

Also the cost and mass-dimensional indexes of DGS and $\mathrm{AE}$ for the hybrid shunting locomotive working in various operating modes have been calculated. It was found out that the second type of hybrid drive is less effective on cost indexes, and in the case of export work it does not satisfy also the mass-dimensional restrictions of shunting locomotive.

By means of traction calculations the comparative analysis of two engines of basic ChME3 and hybrid on its base was made. The calculations were carried out using the program Mathcad complex with train weighing $500 \mathrm{t}$ for export work on a profile Stakhanov-Popasna [11]. What follows from the calculations is that for an almost identical time of movement fuel consumption hybrid of shunting locomotive was made by $126 \mathrm{~kg}$ that is $25 \%$ less, than the basic engine of the ChME3 series. It was found out that the trip efficiency of the modernized locomotive increased from $14,6 \%$ to $19,2 \%$, which confirms the efficiency of introducing a hybrid drive on a shunting locomotive.

\section{Conclusions}

1. Dependences of percent of the general operating time of a locomotive on PLC when performing shunting work are given, export and works in the hill.

2. Taking into account these correspondences for each operating mode $N_{\text {engopt }}$ - the optimum power of DGS, by $\mathrm{kW}$, and $E_{n e}$ - optimum power consumption $\mathrm{AE}, \mathrm{MJ}$ has been calculated. It confirms the fact that for easy shunting work such powerful power plant which is installed on a ChME3 series locomotive is really necessary.

3. Traction calculations have been carried out using the program Mathcad complex for a profile StakhanovPopasna with train weighing $500 \mathrm{t}$ for export work with parameters of the locomotive chosen through results of calculations.

\section{References}

1. P. Wolfs, AUPEC, September 25, 123-123 (2005).

2. C. Akli, Int. Journal of Applied Electromagnetics and Mechanics, 30, 151-162, 2009

3. A. Lohner, IEEE 35th Power Electronics Specialist Conference, 672-676 (2004)

4. H. Yap, IEEE Power Electronics, Machines and Drives Conference, 61-66 (2004)

5. R. Cousineau, IEEE Instrumentation \& Measurement Magazine, February, 25-29 (2006)

6. L. Liudvinavičius, Transport Problems: an International Scientific Journal, 6, 135-142 (2011)

7. A. Falendysh, TEKA. Commission of motorization and energrtics in agriculture, 12, 58-63 (2012)

8. A. Varakin, Transport science and technology, 12, 3440 (2007)

9. E. Kossov, Locomotive inform, 3, 44-45 (2008)

10. M. Sergienko, Selection and scientific substantiation of technical and economic indicators of diesel diesel engines for the locomotive park of Ukraine (Kharkiv, 2000)

11. A. Falendysh, P. Kharlamov, O. Kletska, N. Volodarets, Transportation Research Procedia, 14, 665-671 (2016) 